\title{
Study of the role of Arabidopsis thaliana RNA-polymerase with dual-targeting RPOTmp in plant early development and stress response
}

\author{
Gorbenko I.V.*, Tarasenko V.I., Garnik E.Yu., Belkov V.I., Konstantinov Yu.M., \\ Koulintchenko M.V. \\ Siberian Institute of Plant Physiology and Biochemistry, SB RAS, Irkutsk, Russia \\ *e-mail: gravov.chemistry@gmail.com
}

In a number of dicotyledonous plants, including Arabidopsis, transcription of organellar genes is performed by three nuclear-encoded RNA polymerases, RPOTm, RPOTmp, and RPOTp. RPOTmp is a dual targeting protein, which is presumably involved in gene expression control in both mitochondria and chloroplasts. A previous study of Arabidopsis insertion mutant rpotmp showed that it has retarded growth and development, altered leaf morphology, changed expression of mitochondrial and probably some plastid genes, and decreased activities of the mitochondrial respiratory complexes. To date, the particular importance of RPOTmp as a part of mitochondrial and plastid transcription machinery remains unclear. Despite of the obvious though not fully understood role of RPOTmp in mitochondrial transcription, the function of this NEP polymerase in chloroplasts of dicotyledonous plants is still under discussion. Transcription of plastid genome of lower plants including alga, with the probable exception of Physcomitrella, is performed by PEP-polymerase (Plastid Encoded Plastid) only. Contrastingly, benefits of angiosperms using phage-type NEP-polymerase (and eudicots using even two of these) remain unknown. The aim of this study is to elucidate what role RPOTmp could specifically play in organelles transcription regulation and possibly in retrograde regulation and cell stressresponses. Previously, plant lines overexpressing RPOTmp with either mitochondrial or plastid targeting based on the $A$. thaliana wild-type line as well as lines with RPOTmp complimentary functions based on the rpotmp mutant line were obtained and used for microarray analysis. The transcript level of selected genes isolated from these transgenic plants germinated in different conditions were used for real-time qPCR analysis. It was noticed that some lines with RPOTmp overexpression have increased germination rate under stress condition. We suppose that these lines might have an increased metabolism rate. Also, the bioinformatics analysis of the promoter region of the Arabidopsis rpot 2 gene encoding RPOTmp for different regulatory motifs is presented. 